Supplement of The Cryosphere, 14, 985-1008, 2020

https://doi.org/10.5194/tc-14-985-2020-supplement

(C) Author(s) 2020. This work is distributed under

the Creative Commons Attribution 4.0 License.

(c) (1)

Supplement of

\title{
Twenty-first century ocean forcing of the Greenland ice sheet for modelling of sea level contribution
}

Donald A. Slater et al.

Correspondence to: Donald Slater (daslater@ucsd.edu)

The copyright of individual parts of the supplement might differ from the CC BY 4.0 License. 


\begin{tabular}{|l|l|l|l|l|}
\hline Sector & No. glaciers & $\begin{array}{l}\mathbf{2 0 0 0 - 2 0 1 0} \text { mean sector } \\
\text { ice flux }(\mathbf{G t} / \mathbf{y r})\end{array}$ & $\begin{array}{l}\text { Largest glacier by ice } \\
\text { flux }\end{array}$ & $\begin{array}{l}\text { Largest glacier 2000-2010 } \\
\text { mean ice flux }(\mathbf{G t} / \mathbf{y r})\end{array}$ \\
\hline SE & 47 & 139.0 & Helheim & 25.7 \\
\hline SW & 9 & 17.6 & Kangiata Nunata Sermia & 5.8 \\
\hline CE & 23 & 59.3 & Kangerdlugssuaq & 28.8 \\
\hline CW & 18 & 91.5 & Jakobshavn & 42.7 \\
\hline NE & 25 & 22.4 & Daugaard-Jensen & 9.3 \\
\hline NW & 60 & 95.2 & Kong Oscar & 8.4 \\
\hline NO & 12 & 16.0 & Humboldt & 3.8 \\
\hline
\end{tabular}

Table S1. Statistics of tidewater glaciers in each sector, which play an important role in sector-by-sector retreat projections. Note that the largest glacier by ice flux excludes glaciers with floating ice tongues.

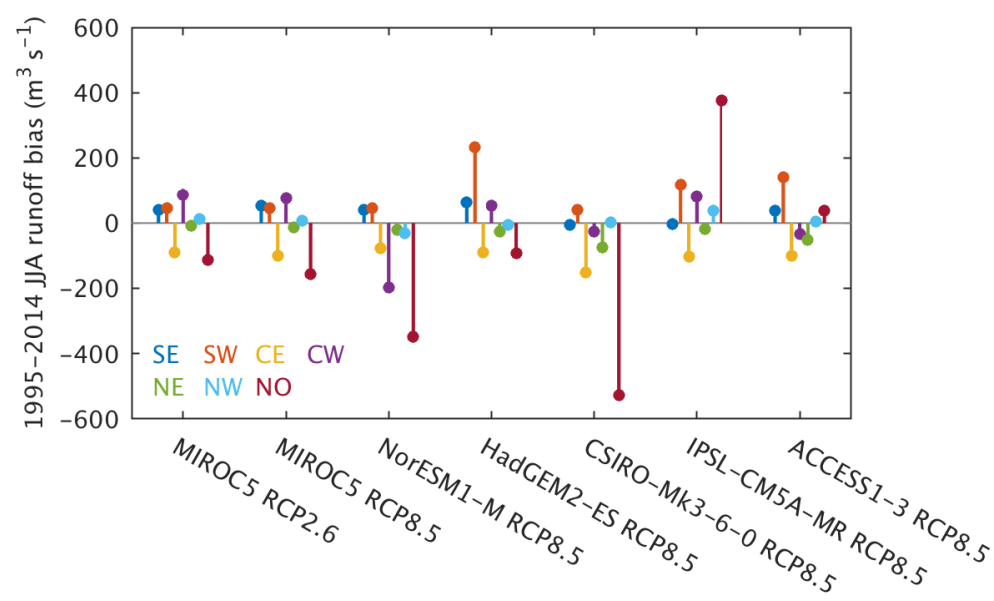

Figure S1. June-July-August (JJA) runoff biases for the glacier with the largest 2000-2010 ice flux per sector (excepting glaciers with permanent ice shelves). A positive value indicates that MAR forced by the CMIP5 AOGCM simulates more runoff over the period 19952014 than RACMO2.3p2 forced by ERA-Interim. For example, at Helheim Glacier in SE Greenland, the JJA runoff from MAR forced by MIROC5 RCP8.5 is $55 \mathrm{~m}^{3} \mathrm{~s}^{-1}$ larger than RACMO2.3p2 forced by ERA-Interim over the period 1995-2014, as illustrated in Fig. 2c of the main article. 

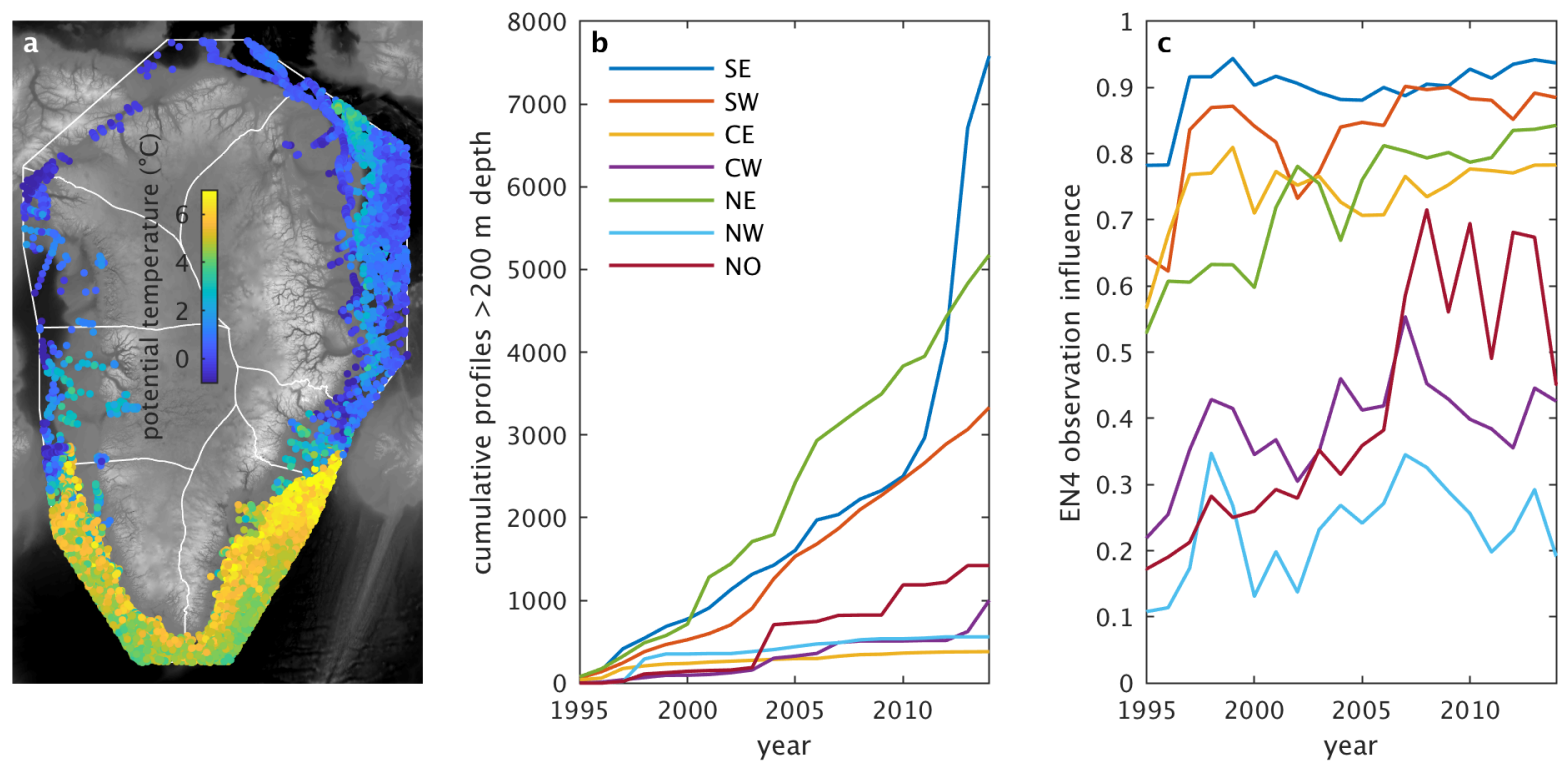

Figure S2. Oceanographic profiles underlying the EN4 gridded product we use to perform the bias correction. (a) Potential temperature at $200 \mathrm{~m}$ depth for all profiles reaching a depth greater than $200 \mathrm{~m}$ during the period 1995-2014. The white lines show the sector boundaries described in the main article. (b) Cumulative number of profiles per sector during the same time period. (c) Observation influence for the EN4 gridded product as a function of time. Observation influence is a metric provided with the EN4 product to quantify the extent to which gridded values are influenced by observations that are close in time or space. A value close to 0 indicates there is little profile data to constrain the gridded value while a value close to 1 indicates the gridded value is tightly constrained by observations.

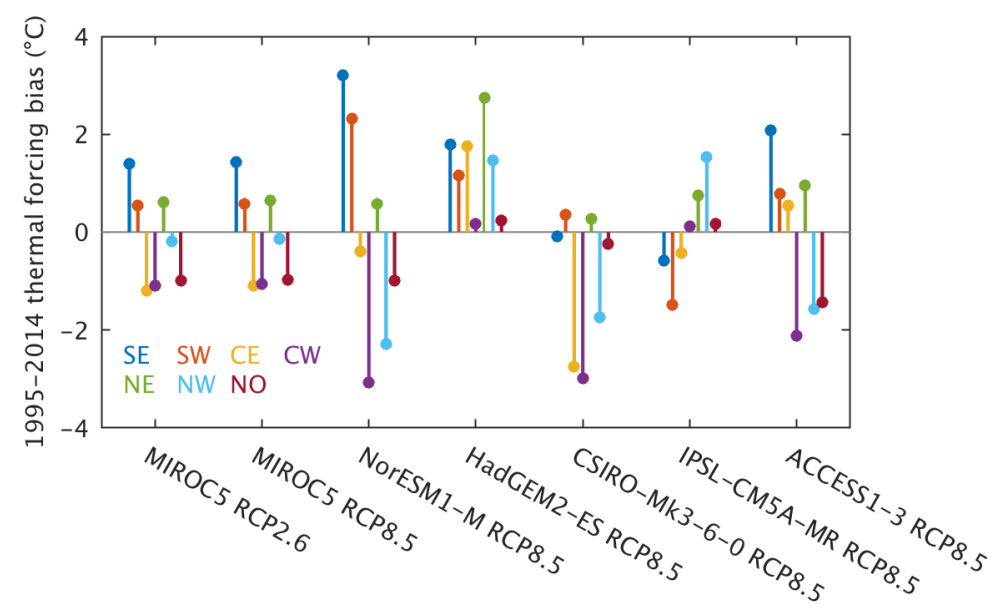

Figure S3. Thermal forcing biases per ice ocean sector for each CMIP AOGCM. A positive value indicates that the CMIP AOGCM is warmer than the observations (EN4) over the 1995-2014 period. For example, for MIROC5 RCP8.5, the SE region is $1.4^{\circ} \mathrm{C}$ warmer than EN4, as illustrated in Fig. 3c of the main article. 

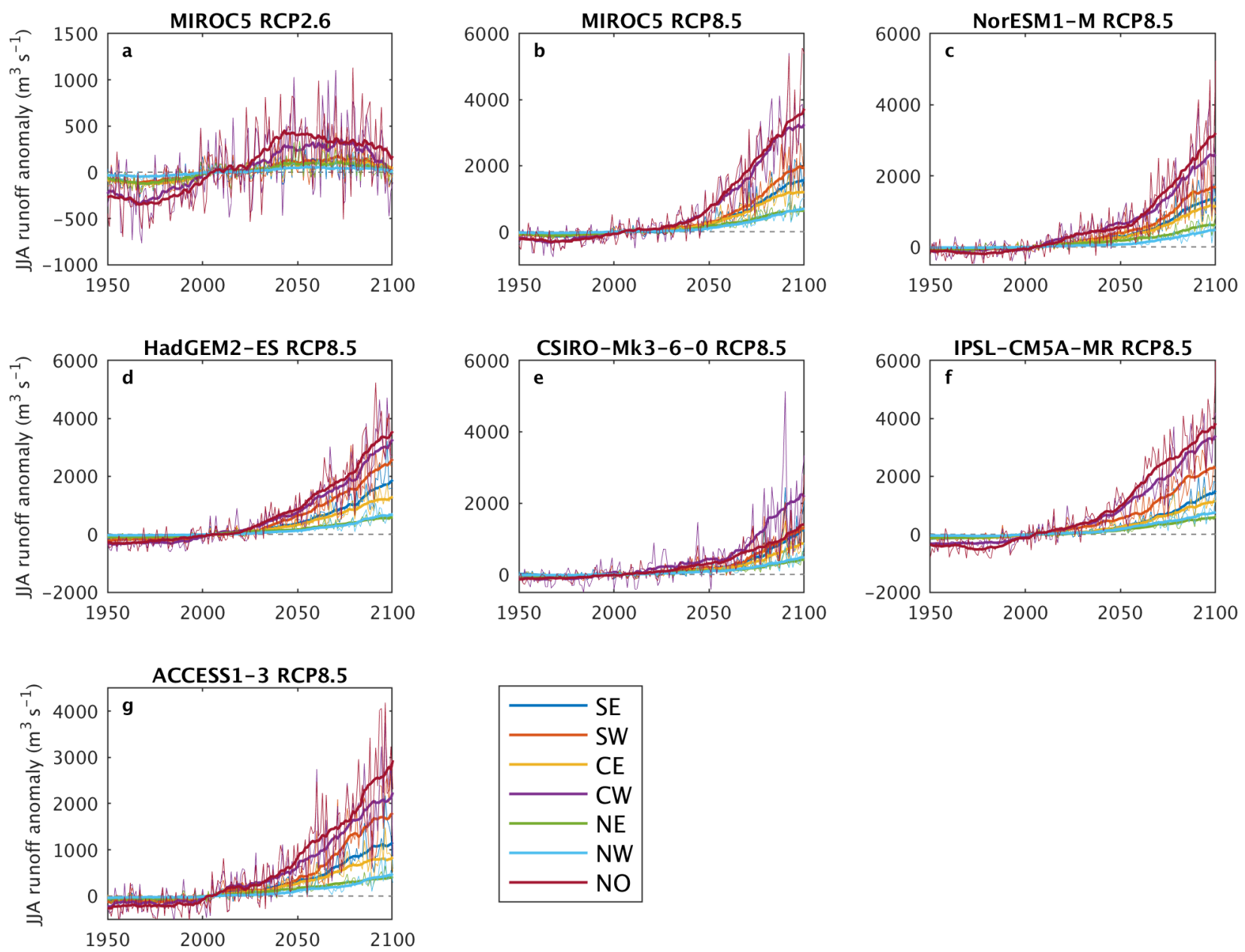

Figure S4. Evolution of JJA runoff in all models and sectors, grouped by CMIP5 AOGCM. Each plot shows a CMIP5 AOGCM, while the colors on each plot differentiate the ice-ocean sectors. For each ice-ocean sector we plot runoff for the largest glacier by ice flux in the sector, as in Fig. 7 of the main paper. 

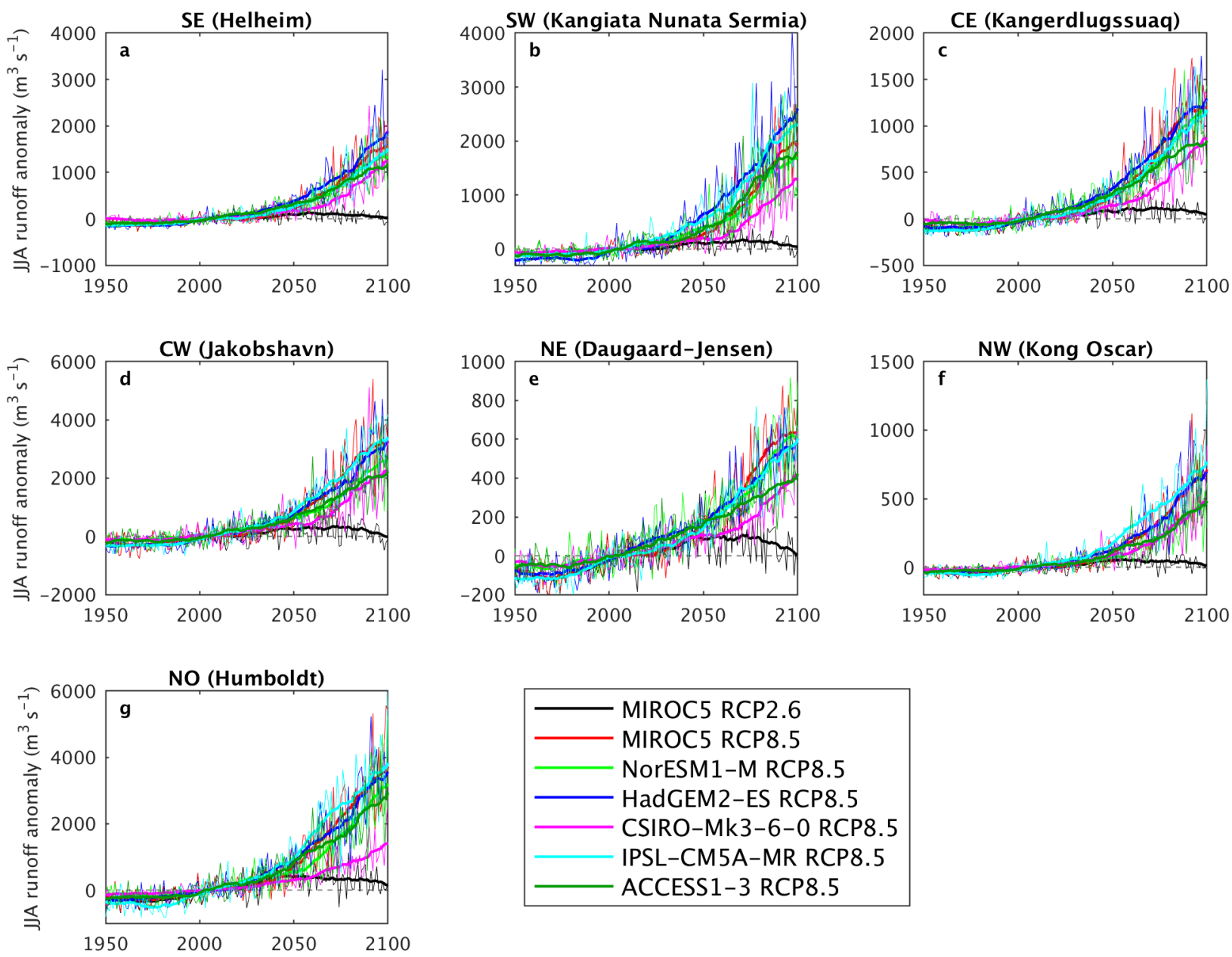

Figure S5. Evolution of JJA runoff in all models and sectors, grouped by ice-ocean sector. Each plot shows runoff for the largest glacier by ice flux in each sector (Table S1), while the colors differentiate the CMIP5 AOGCMs. For clarity, annual values are thin lines and a 20-year moving mean is plotted in thick lines. 

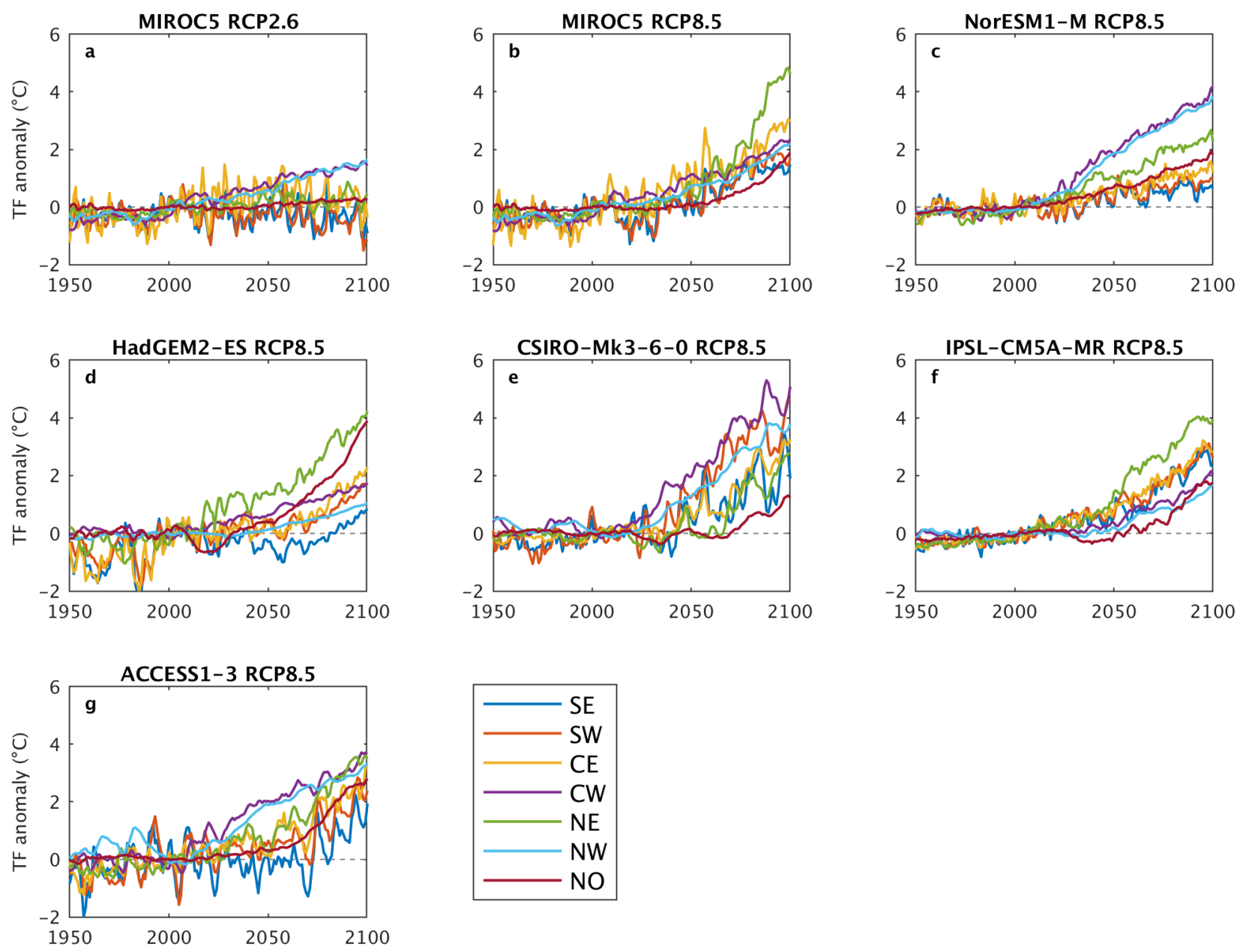

Figure S6. Evolution of 200-500 m ocean thermal forcing in all models and sectors, grouped by CMIP5 AOGCM. Each plot shows a CMIP5 AOGCM, while the colors on each plot differentiate the ice-ocean sectors. 

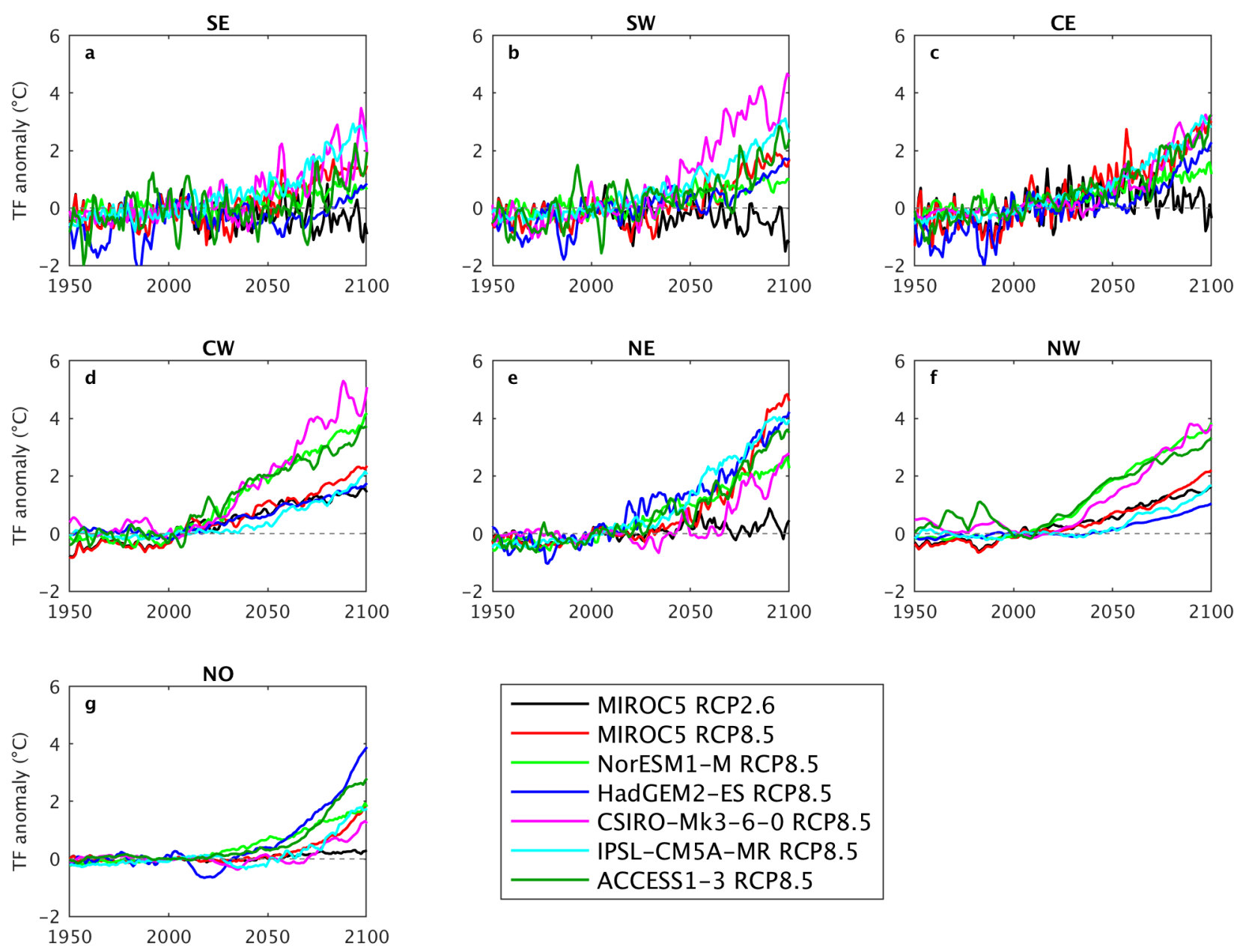

Figure S7. Evolution of 200-500 m ocean thermal forcing in all models and sectors, grouped by ice-ocean sector. Each plot shows an ice-ocean sector while the colors differentiate the CMIP5 AOGCMs. 

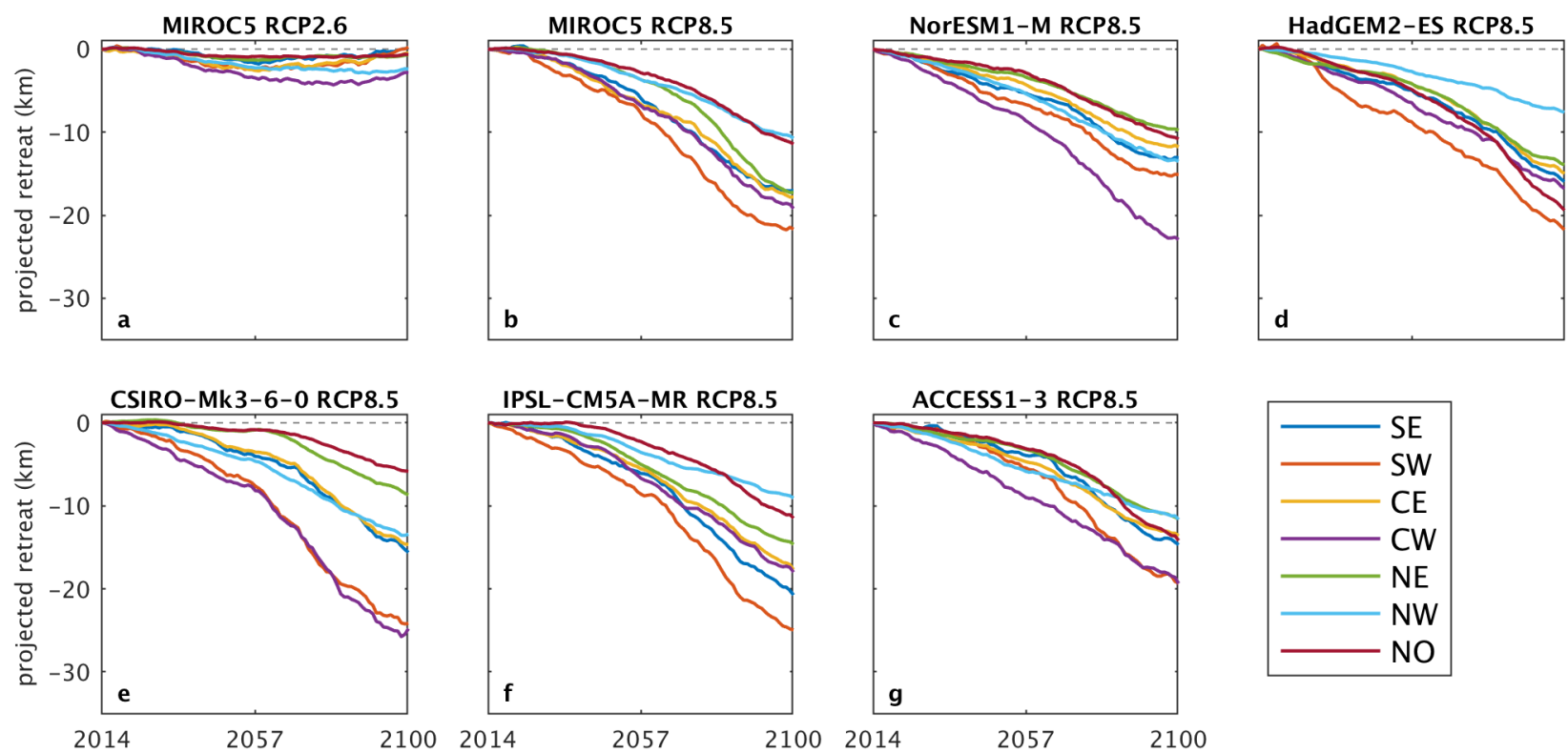

Figure S8. Retreat projections grouped by CMIP5 AOGCM. Only the medium retreat cases are shown.
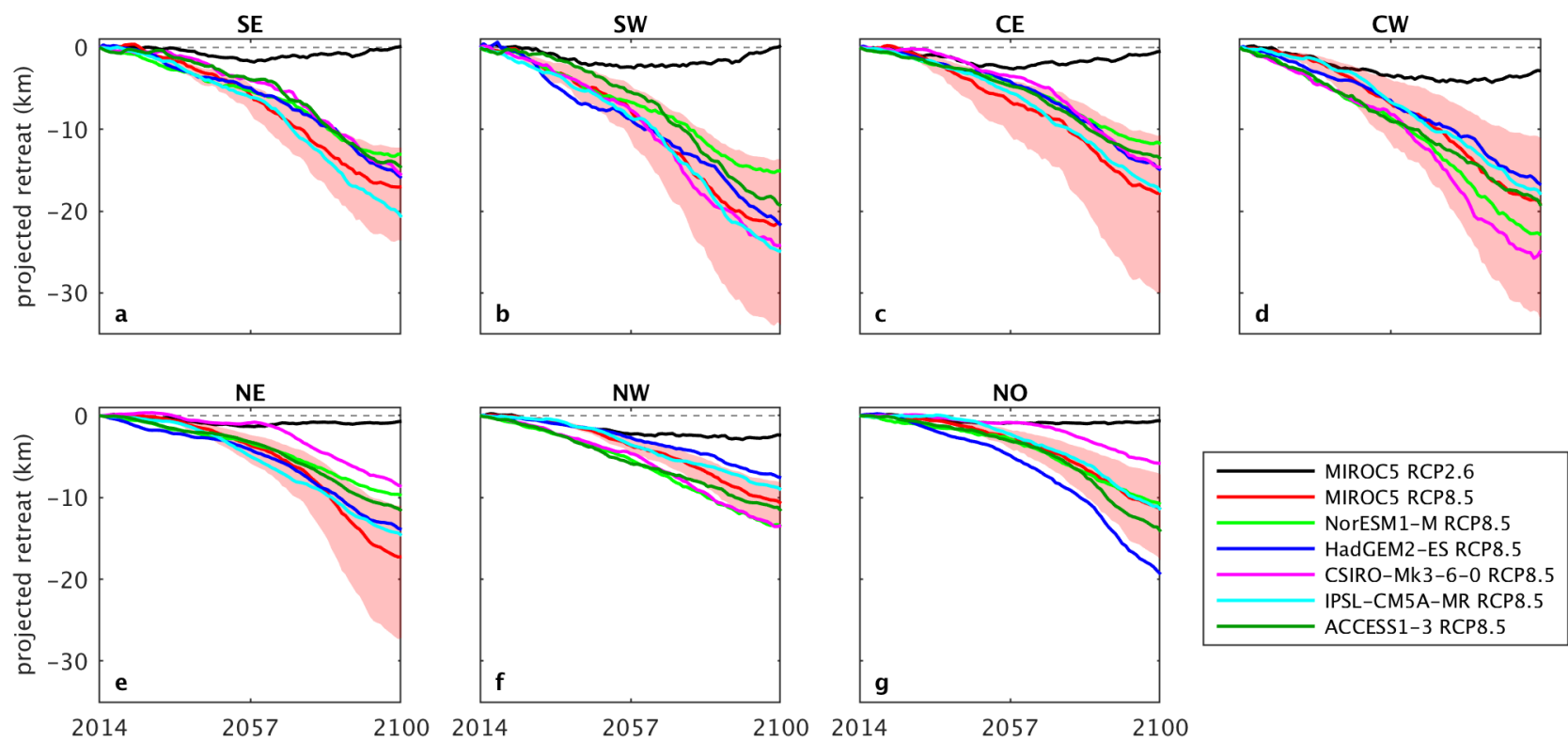

Figure S9. Retreat projections grouped by sector. The lines show the medium retreat projections for each CMIP AOGCM while the shading shows the low and high projections for MIROC5 RCP8.5 only; the low to high range is similar for all other RCP8.5 simulations. 

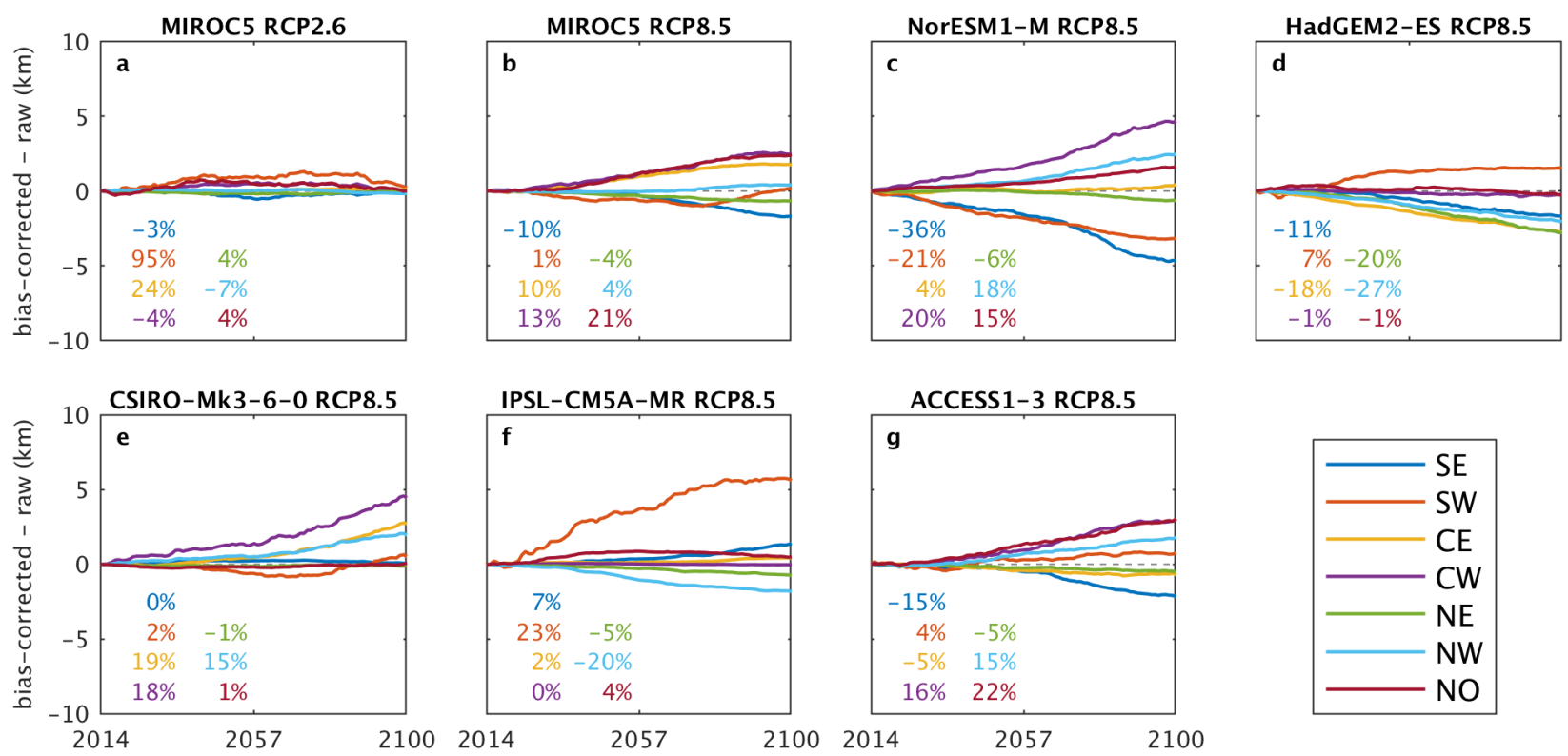

Figure S10. Impact of runoff and thermal forcing bias correction on retreat projections. Shown is the difference between the bias-corrected retreat projection and the projection without any bias correction. The coloured numbers show the relative increase in retreat by 2100 in each sector resulting from the bias correction. Given that the form of the retreat and melt rate parameterisations (Eqs. 1 and 2 of the main article) are similar, the coloured numbers also give a sense of how sensitive projections in the submarine melt formulation are to the bias correction. 